\title{
ICLP 2010 \\ A NEW COMPREHENSIVE LIGHTNING INSTRUMENTATION SYSTEM FOR PAD 39B AT THE KENNEDY SPACE CENTER, FLORIDA
}

\author{
Carlos T. Mata Vladimir A. Rakov Angel G. Mata Tatiana Bonilla Emmanuel Navedo \\ ASRC Aerospace University of Florida ASRC Aerospace ASRC Aerospace ASRC Aerospace \\ Carlos.T.Mata@nasa.gov Rakov@ece.ufl.edu Angel.Mata@alts-inc.com Tatiana.Bonilla-1@nasa.gov Emmanuel.Navedo-1@nasa.gov
}

\begin{abstract}
A new comprehensive lightning instrumentation system has been designed for Launch Complex 39B at the Kennedy Space Center, Florida. This new instrumentation system includes the synchronized recording of six high-speed video cameras, currents through the nine downconductors of the new lightning protection system, four B-dot, 3-axis measurement stations, and five D-dot stations composed of two antennas each. The instrumentation system is composed of centralized transient recorders and digitizers that located close to the sensors in the field. The sensors and transient recorders communicate via optical fiber. The transient recorders are triggered by the B-dot sensors, the E-dot sensors, or the current through the downlead conductors. The high-speed cameras are triggered by the transient recorders when the latter perceives a qualified trigger.
\end{abstract}

\section{INTRODUCTION}

The Space Shuttle vehicle lightning protection system at Launch Complex 39B (LC39) consisted of a catenary wire running north to south, with an insulating mast providing support in the middle. This system was built to support the Shuttle Program and has existed since the first launch, STS-1, on April 12, 1981. A new lightning protection system was designed and its construction began in 2007. The new lightning protection system consists of three towers supporting a catenary wire system with a total of nine downleads (see Figure 1). During 2009, the Shuttle lightning protection system was removed after the three towers of the new lightning protection system were erected. A temporary catenary wire was installed between lightning protection towers 1 and 2 (northwest and northeast towers, respectively) to protect the standby rescue Shuttle during the Hubble Repair Mission and the Ares I-X test vehicle. In December 2009, the new catenary wire array was installed. The new three-tower lightning protection system is described in [1].

The Shuttle lightning protection system was instrumented with Pearson Coils to measure the current to ground at the two downleads. The analog signals from the Pearson Coils were transmitted through coaxial cables to transient recorders located underneath the pad. These runs were approximately $300 \mathrm{~m}$ each. The current measurements were used to determine when the lightning protection system was struck by lightning and to estimate the severity of the strike. This instrumentation suffered from severe deficiencies because of the extremely long coaxial-cable runs between the Pearson Coils and the transient recorders, the condition of the coaxial cables, and the obsolescence of the transient recorders.

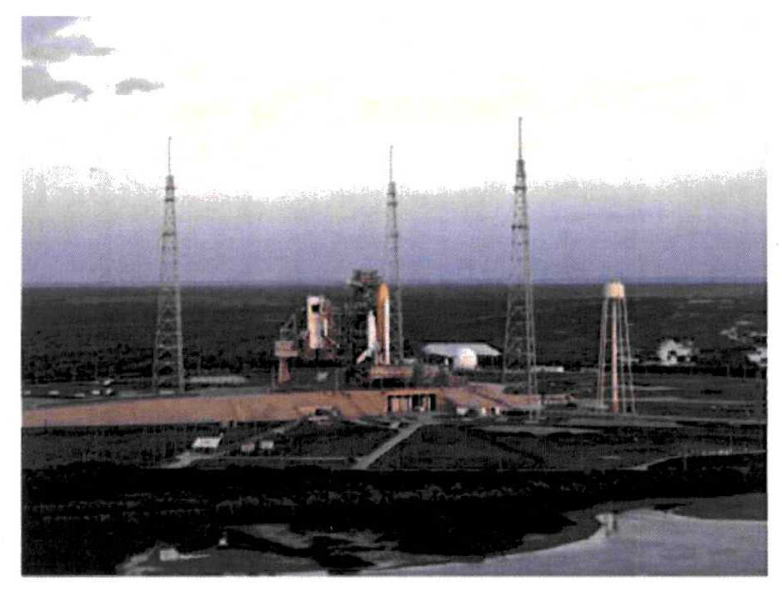

Figure 1. New lightning protection at LC39B, Kennedy Space Center, Florida, showing the standby rescue Shuttle during the Hubble repair mission in 2009.

New lightning instrumentation has been designed for the new LC39B lightning protection system to overcome the deficiencies of the previous lightning instrumentation system and to provide much more capabilities. This instrumentation system is expected to be installed during 2011 and 2012. A subset of this lightning instrumentation system has been deployed and tested at the International Center for Lightning Research and Testing (ICLRT), Camp Blanding, Florida.

\section{INSTRUMENTATION SYSTEM}

The instrumentation system is composed of transient recorders, remote digitizers, $\mathrm{dB} / \mathrm{dt}$ sensors, $\mathrm{dE} / \mathrm{dt}$ sensors, 
current sensors, and high-speed cameras.

\subsection{Transient Recorders}

The three transient recorders are state-of-the-art GEN16t models (formerly manufactured by Nicolet but now manufactured by HBM) with master/slave interconnection, providing timing synchronization of 100 ns or better between transient recorders (see Figure 2). The GEN16t has 16 slots, one of which is used by the master/slave card, leaving 15 slots to install the fiberoptic receiver cards, each of which can accommodate up to four remote digitizers. This arrangement provides scalable data acquisition with an original capability of 180 channels. The fiber-optic receiver cards are designed to communicate with the remove digitizers, which provide A/D conversion close to the sensor in the field. The GEN16t provides a large array of triggering options. The system can be configured to trigger in window mode (when a measurement leaves a predefined window) from any of its channels. If a GEN16t triggers, it automatically triggers the other GEN16t connected to the master/slave bus. The Gen $16 \mathrm{t}$ is capable of recording at a maximum rate of 100 megasamples per second per channel.

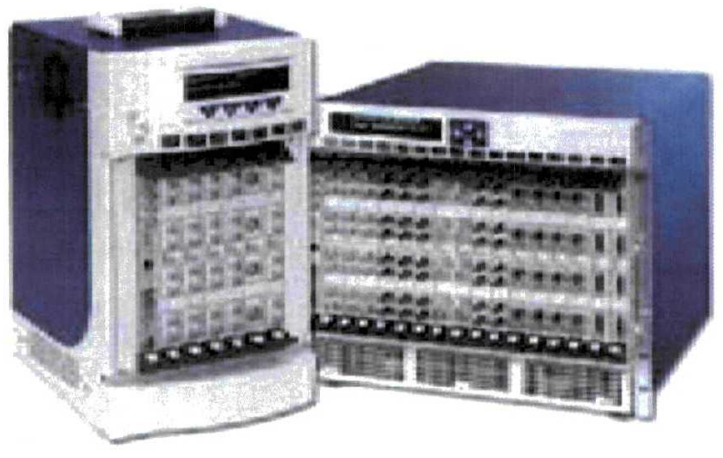

Figure 2. GEN7t 7-slot tower (left) and GEN16t 16-slot rack-mount chassis.

\subsubsection{Master/Slave Synchronization}

Although the name "master/slave" may be misleading, this optional card offers power timing synchronization among the GEN16t transient recorders connected to the master/slave bus. With this option, all transient recorders are time-synchronized to better than $100 \mathrm{~ns}$. Also, this card allows any GEN16t to trigger any other GEN16t connected to the bus.

\subsubsection{Fiber-Optic Receiver Card}

The fiber-optic receiver card can accommodate up to four channels, with an onboard memory of 900 megasamples to be used by the enabled channels. The fiber-optic link is a single-mode fiber with automatic fiber delay compensation for fibers up to $4 \mathrm{~km}$ in length. This card is advertised to work with up to $12 \mathrm{~km}$, lowloss, single-mode fibers.

\subsubsection{Isolated Digitizer 7600}

These are ruggedized digitizers with a sampling rate of 100 megasamples per second. The digitizers have an open collector output that can be turned on or off remotely from the transient recorder. The digitizers are powered by a lead acid battery, through a battery charger powered by facility power. The facility power passes through a normally closed relay, which is commanded to disconnect the facility power when the presence of lightning is imminent. The relay is operated by the open collector output of the digitizers. The digitizers are placed as close as possible to the sensors in the field and they are housed in stainless steel 316 EMI enclosures.

\subsection{Sensors}

\subsubsection{Pearson Electronics Current Monitor}

Pearson Electronics Current Monitors, model 1330, are used to measure the current to ground at each of the nine downleads of the lightning protection system. The sensors have a sensitivity of $0.005 \mathrm{~V} / \mathrm{A}$, maximum current rating of $100 \mathrm{kA}$, and a low and high cut-off frequency of $0.9 \mathrm{~Hz}$ and $1.5 \mathrm{MHz}$, respectively. This same current monitor model was used in 2009 to measure the channel current of some of the triggered events at the ICLRT. If lightning strikes the catenary wire system, these sensors will help in determining the strike intensity and location.

\subsection{2 $d B / d t$ Sensors}

Each of the four $\mathrm{dB} / \mathrm{dt}$ stations in the perimeter of LC39B is instrumented with three EG\&G B-dot sensors, model MGL-2, with a frequency response greater than 300 $\mathrm{MHz}$, an equivalent area of $1 \times 10^{-2} \mathrm{~m}^{2}$, and a maximum field change of $2 \times 10^{4} \mathrm{~T} / \mathrm{s}$. The three sensors per station are arranged in an orthogonal configuration, with dedicated digitizers for each one of the sensors. These sensors will serve as direction finders and will help in determining the intensity of the strike. Also, a differential-time-of-arrival technique will be used to help locate the attachment point.

\subsection{3 dE/dt Sensors}

Each of the five $\mathrm{dE} / \mathrm{dt}$ stations in the perimeter of LC39B is instrumented with two EG\&G D-dot sensors, model ACD-4(R), with a frequency response greater than 1.0 $\mathrm{GHz}$ and an equivalent area of $1 \times 10^{-2} \mathrm{~m}^{2}$. A differentialtime-of-arrival technique is used to locate the attachment point. 


\subsection{High-Speed Video Cameras}

Two high-speed video cameras, Vision Research Phantom v310, will be used on each tower, for a total of six high-speed video cameras. The Phantom v310 can sample up to $3,250 \mathrm{fps}$ at its maximum resolution of $1280 \times 800$, with a selectable pixel bit depth of 12 bits, segmented memory, IRIG-B synchronization, and external trigger input. The Phantom v310 cameras are triggered from the transient recorder trigger output.

\section{CONCEPT OF OPERATION}

The GEZN 16t transient recorders are configured to trigger when either D-dot sensor exceeds $5 \mathrm{kV} / \mathrm{m} / \mu \mathrm{s}$ or the current through the downleads exceeds $500 \mathrm{~A}$. The GEN16t transient recorders are configured to record an infinite number of $30 \mathrm{~ms}$ sweeps (continuous recording) with a pretrigger of $20 \mathrm{~ms}$. The sweep stretch feature is enabled, which means that if during the posttrigger window a qualified trigger is encountered, the posttrigger window will restart. The automatic-export feature per sweep is also enabled, which allows the operators to review the data remotely without interrupting the acquisition.

The $45^{\text {th }}$ Weather Squadron provides weather forecasting services to the Kennedy Space Center. Forecasting services include the issuance of a warning when lightning is imminent. When this warning is issued, the Phantom v310 cameras are armed and the GEN16t transient recorders command all the digitizers to switch out the facility power (using their open collector TTL outputs). All the digitizers remain under battery power for the duration of the storm. The GEN16t transient recorders remain armed $24 / 7$.

The Phantom v310 analog output is monitored at all times and recorded using digital video recorders (DVRs), which are also triggered by the GEN16t transient recorders. The DVR has a 1 -minute circular buffer with a $50 \%$ pretrigger. The Phantom $\mathrm{v} 310$ cameras are configured in segmented-memory mode and they download each segment as data is gathered, while continuing to record if a qualified trigger is received.

A differential-time-of-arrival technique is used with the B-dot and D-dot sensors to locate the attachment point. The downlead currents are used to estimate the intensity of the strike. The Phantom v310 cameras are used to locate the attachment point.

\section{CONCLUSIONS}

A comprehensive lightning instrumentation system is presented for the new lightning protection system at LC39B at the Kennedy Space Center, Florida. The instrumentation system uses state-of-the-art transient recorders monitoring a multitude of current, B-dot, and D-dot sensors at various points of LC39B to estimate the strike intensity and the attachment point. High-speed video cameras are also used to monitor most of LC39B and to locate the attachment point.

\section{REFERENCES}

[1] C. T. Mata, V. A. Rakov, "Evaluation of lightning incidence to elements of a complex structure: a Monte Carlo approach," International Conference on Grounding and Earthing \& $3^{\text {rd }}$ International Conference on Lightning Physics and Effects (Ground '2008; $3^{\text {rd }}$ LPE), Florianopolis, Brazil, November 2008. 


\title{
ICLP 2010 \\ A NEW COMPREHENSIVE LIGHTNING INSTRUMENTATION SYSTEM FOR PAD 39B AT THE KENNEDY SPACE CENTER, FLORIDA
}

\author{
Carlos T. Mata \\ Vladimir A. Rakov \\ Tatiana Bonilla \\ ASRC Aerospace \\ Carlos.T.Mata@nasa.gov \\ University of Florida \\ Rakov@ece.ufl.edu \\ ASRC Aerospace \\ Tatiana.Bonilla-1@nasa.gov \\ Angel G. Mata \\ Emmanuel Navedo \\ Gary P. Snyder \\ ASRC Aerospace \\ ASRC Aerospace \\ NASA, KSC \\ angel.mata@alts-inc.com Emmanuel.Navedo-1@nasa.gov Gary.P.Snyder@nasa.gov
}

\begin{abstract}
A new comprehensive lightning instrumentation system has been designed for Launch Complex 39B at the Kennedy Space Center, Florida. This new instrumentation system includes the synchronized recording of six high-speed video cameras; currents through the nine downconductors of the new lightning protection system; four $\mathrm{dH} / \mathrm{dt}, 3$-axis measurement stations; and five $\mathrm{dE} / \mathrm{dt}$ stations composed of two antennas each. The instrumentation system is composed of centralized transient recorders and digitizers located close to the sensors in the field. The sensors and transient recorders communicate via optical fiber. Sensor outputs are sampled by fiber-optic digitizers, which transmit the digitized data to transient recorders via fiber optics. The transient recorders are triggered by the $B$-dot sensors, the $\mathrm{dE} / \mathrm{dt}$ sensors, or the current through the downlead conductors. The high-speed cameras are triggered by the trigger output of the transient recorder when the recorder perceives a qualified trigger.
\end{abstract}

\section{INTRODUCTION}

The Space Shuttle vehicle lightning protection system at Launch Complex 39B (LC39) consisted of a catenary wire running north to south, with an insulating mast providing support in the middle. This system was built to support the Shuttle Program and has existed since the first launch, STS-1, on April 12, 1981. A new lightning protection system was designed and its construction began in 2007. The new lightning protection system consists of three towers supporting a catenary wire system with a total of nine downleads (see Figure 1). In 2009, the Shuttle lightning protection system was removed after the three towers of the new lightning protection system were erected. A temporary catenary wire was installed between lightning protection towers 1 and 2 (northwest and northeast towers, respectively) to protect the standby rescue Shuttle during the Hubble repair mission and the Ares I-X test flight. In December
2009 , the new catenary wire array was installed. The new three-tower lightning protection system is described in [1].

The Shuttle lightning protection system was instrumented with Pearson Coils to measure the current to ground at the two downleads. The analog signals from the Pearson Coils were transmitted through coaxial cables to transient recorders located underneath the pad. These runs were approximately $300 \mathrm{~m}$ each. The current measurements were used to determine when the lightning protection system was struck by lightning and to estimate the severity of the strike. This instrumentation suffered from severe deficiencies because of the extremely long coaxial-cable runs between the Pearson Coils and the transient recorders, the condition of the coaxial cables, and the obsolescence of the transient recorders.

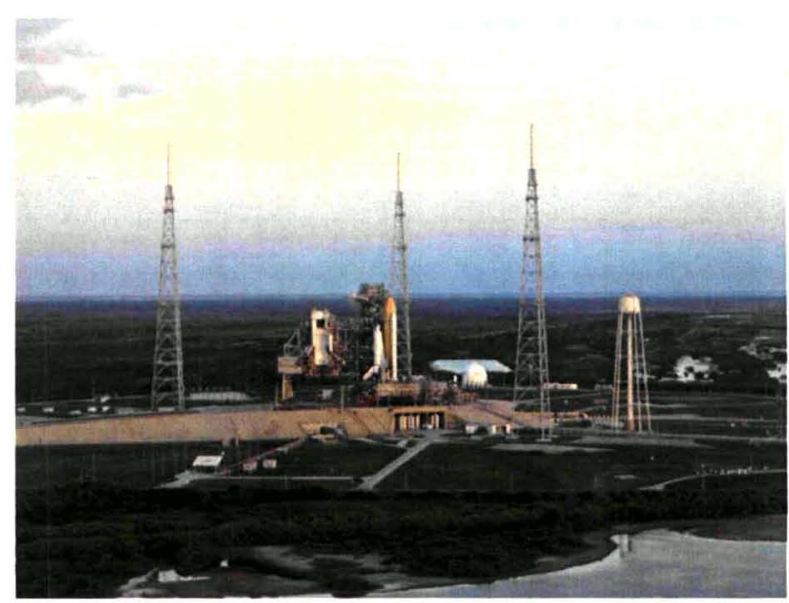

Figure 1. New lightning protection at LC39B, Kennedy Space Center, Florida, showing the standby rescue Shuttle during the Hubble repair mission in 2009. 
New lightning instrumentation has been designed for the new LC39B lightning protection system to overcome the deficiencies of the previous lightning instrumentation system and to provide much more capabilities. This instrumentation system is expected to be installed by September 2010. A subset of this lightning instrumentation system has been deployed and tested at the International Center for Lightning Research and Testing (ICLRT), Camp Blanding, Florida. This article describes only the portion of the lightning instrumentation subset installed at LC39B, not the subset installed in the mobile launcher tower, which provides transient instrumentation capabilities to the mobile launcher tower itself and the vehicle.

\section{INSTRUMENTATION SYSTEM}

The instrumentation system is composed of transient recorders, remote digitizers, $\mathrm{dH} / \mathrm{dt}$ sensors, $\mathrm{dE} / \mathrm{dt}$ sensors, current sensors, and high-speed cameras.

\subsection{Transient Recorders}

There are three state-of-the-art transient recorders, GEN16t models (formerly manufactured by Nicolet but now manufactured by HBM) with master/slave interconnection, providing timing synchronization of $100 \mathrm{~ns}$ or better between transient recorder channels (see Figure 2). The GEN16t has 16 slots, one of which is used by the master/slave card, leaving 15 slots for installation of the fiber-optic receiver cards, each of which can accommodate up to four remote digitizers. This arrangement provides scalable data acquisition with an original capability of 180 channels. The fiber-optic receiver cards are designed to communicate with the remote digitizers, which provide $\mathrm{A} / \mathrm{D}$ conversion close to the sensor in the field. The GEN16t provides a large array of triggering options. The system can be configured to trigger in dual-threshold mode (when a measurement exceeds predefined thresholds) from any of its channels. If a GEN16t triggers, it automatically triggers the other GEN16t connected to the master/slave bus. The Gen16t is capable of recording at a maximum rate of 100 megasamples per second per channel.

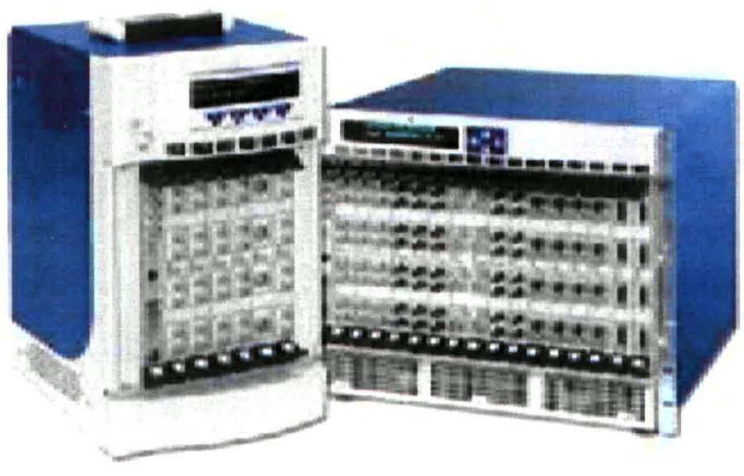

Figure 2. GEN7t 7-slot tower (left) and GEN16t 16-slot rack-mount chassis.

\subsubsection{Master/Slave Synchronization}

Although the name "master/slave" may be misleading, this optional card offers power timing synchronization among the GEN16t transient recorders connected to the master/slave bus. With this option, all transient recorders are time-synchronized to better than $100 \mathrm{~ns}$. Also, this card allows any GEN16t to trigger any other GEN16t connected to the triggering bus.

\subsubsection{Fiber-Optic Receiver Card}

The fiber-optic receiver card can accommodate up to four channels, with an onboard memory of 900 megasamples to be used by the enabled channels. The fiber-optic link is a single-mode fiber with automatic fiber delay compensation for fibers up to $4 \mathrm{~km}$ in length. This card is advertised to work with up to $12 \mathrm{~km}$, lowloss, single-mode fibers.

\subsubsection{Isolated Digitizer 7600}

These are ruggedized digitizers with a sampling rate of 100 megasamples per second. The digitizers (Figure 3) have an open collector output that can be turned on or off remotely from the transient recorder. The digitizers are powered by a lead acid battery, through a battery charger, which receives its power from facility power. The facility power passes through a normally closed relay, which is commanded to disconnect the battery charger from facility power when the presence of lightning is imminent (this is referred to as a Phase II warning, which is a service provided by the 45 th Weather Squadron at Patrick Air Force Station). The relay is operated by the open collector output of the digitizers. The digitizers are placed as close as possible to the sensors in the field and they are housed in stainless-steel 316 EMI enclosures.

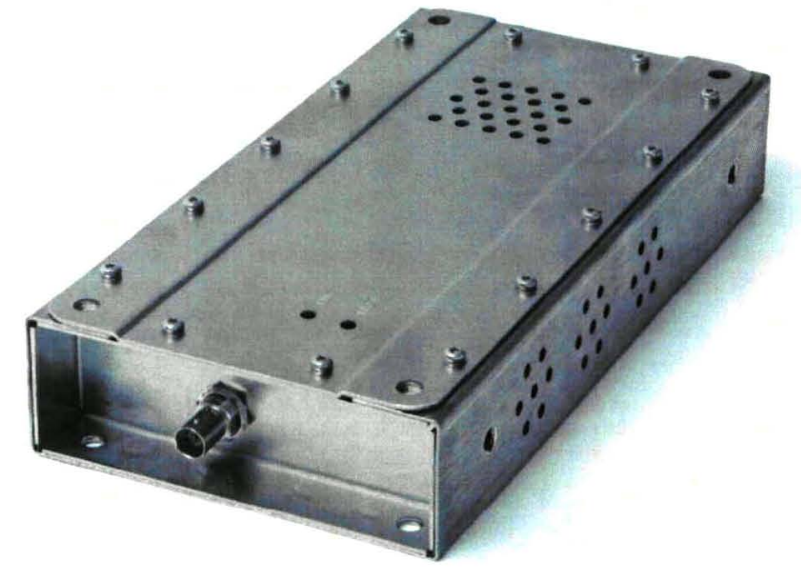

Figure 3. Isolated Digitizer 7600. On this image, only the input signal BNC connector is visible. Power, fiber optic and open collector connectors are on the back panel. 


\subsection{Sensors}

\subsubsection{Pearson Electronics Current Monitor}

Pearson Electronics Current Monitors, model 1330, are used to measure the current to ground at each of the nine downleads of the lightning protection system. The sensors have a sensitivity of $0.005 \mathrm{~V} / \mathrm{A}$, maximum current rating of $100 \mathrm{kA}$, and a low and high cut-off frequency of $0.9 \mathrm{~Hz}$ and $1.5 \mathrm{MHz}$, respectively. This same current monitor model was used in 2009 to measure the channel current of some of the triggered events at the ICLRT. If lightning strikes the catenary wire system, these sensors will help in determining the strike intensity and location.

\subsubsection{B-dot Sensors}

Each of the four $\mathrm{dH} / \mathrm{dt}$ stations in the perimeter of LC39B is instrumented with three EG\&G B-dot (free-field) sensors, model MGL-2 (Figure 4), with a frequency response greater than $300 \mathrm{MHz}$, an equivalent area of $1 \times 10^{-2} \mathrm{~m}^{2}$, a rise time less than $1.2 \mathrm{~ns}$, maximum output (peak) of $5 \mathrm{kV}$, and a maximum field change of $2 \times 10^{4} \mathrm{~T} / \mathrm{s}$. The output connector of each sensor is TCC (100-ohm twin axial).

The three sensors per station are arranged in an orthogonal configuration, with dedicated digitizers for each of the sensors. These sensors serve as direction finders and help in determining the intensity of the strike. Also, a differential-time-of-arrival technique is used to help locate the attachment point.

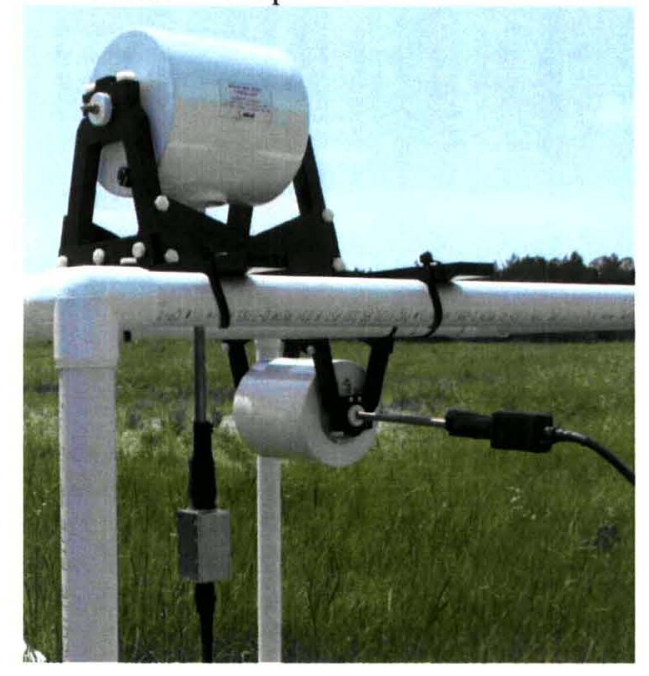

Figure 4. EG\&G B-dot radial and axial sensors (MGL-2(R) top and MGL-2(A) bottom, respectively), each with an EG\&G balun (DMB-7E).

\subsubsection{D-dot Sensors}

Each of the five dE/dt stations in the perimeter of LC39B is instrumented with two EG\&G D-dot (free-field) sensors, model ACD-4(R) (Figure 5), with a frequency response greater than $1.0 \mathrm{GHz}$ and an equivalent area of $1 \times 10^{-2} \mathrm{~m}^{2}$, a rise time less than $0.33 \mathrm{~ns}$ and a maximum output of $5 \mathrm{kV} .{ }^{1}$ The output connector of each sensor is TCC (100-ohm twin axial).

A differential-time-of-arrival technique is used to locate the attachment point.

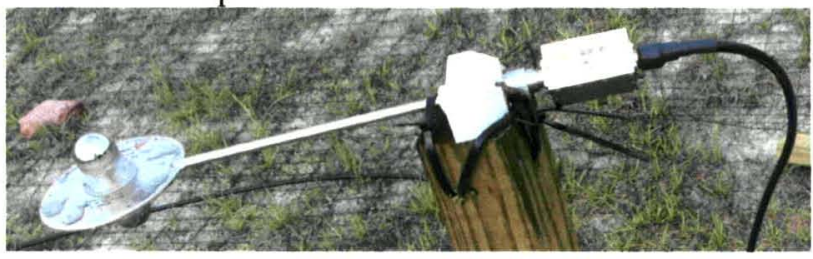

Figure 5. EG\&G D-dot sensor (ACD-4C(R)) with an EG\&G balun (DMB-7E).

\subsubsection{Balun}

The differential-mode balun is used to transform the balanced 100 -ohm signal from a differential (free-field) sensor to an unbalanced 50 -ohm signal for input to a 50 -ohm coaxial cable, oscilloscope, or isolated data link. It changes the input signal of Vo across $100 \mathrm{ohms}$ to an output signal of $\mathrm{Vo} / 2$ across $50 \mathrm{ohms}$; half of the input power is dissipated in the balun so that the insertion loss is $3 \mathrm{~dB}$.

\subsection{High-Speed Video Cameras}

Two high-speed video cameras, Vision Research Phantom v310 (Figure 6), are used on level E of each lightning protection tower, for a total of six high-speed video cameras. The Phantom v310 can sample up to $3,250 \mathrm{fps}$ at its maximum resolution of $1280 \times 800$, with a selectable pixel bit depth of 12 bits, segmented memory, IRIG-B synchronization, and external trigger input. The Phantom v310 cameras are triggered from the transientrecorder trigger output.

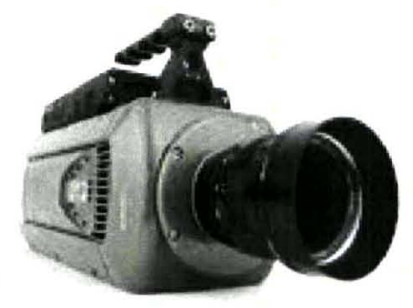

Figure 6. Vision Research's Phantom v310 camera.

The Phantom v310 cameras are operated in segmentedmemory mode with continuous recording enabled, which

\footnotetext{
${ }^{1}$ When filled with $\mathrm{SF}_{6} ; 2 \mathrm{kV}$ otherwise.
} 
allows the camera to start downloading each segment after each trigger is received, while continuing to record video to the next segment. Using this operating mode allows for $24 / 7$ operation, with the risk of losing data only if all the segments are full before the download of any particular segment is finished.

\section{CONCEPT OF OPERATION}

The GEN16t transient recorders are configured to trigger when either $D$-dot sensor exceeds $4 \mathrm{kV} / \mathrm{m} / \mu \mathrm{s}$ or the current through any of the downleads exceeds $500 \mathrm{~A}$. The GEN16t transient recorders are configured to record an infinite number of $30 \mathrm{~ms}$ sweeps (continuous recording) with a pretrigger of $20 \mathrm{~ms}$. The sweep stretch feature is enabled, which means that the posttrigger window will restart if it encounters a qualified trigger. The automatic-export feature per sweep is also enabled, which allows the operators to review the data remotely without interrupting the acquisition. The GEN16t requires computer communication and works with a FIFO memory, so that recorded segments are downloaded to the computer and memory becomes available in the GEN16t as data is transferred.

The $45^{\text {th }}$ Weather Squadron provides weather forecasting services to the Kennedy Space Center. Forecasting services include the issuance of a warning when lightning is imminent. When this warning is issued, the Phantom v310 cameras are armed and the GEN16t transient recorders command all the digitizers to switch out the facility power (using their open collector TTL outputs). All the digitizers remain under battery power for the duration of the storm. The GEN16t transient recorders remain armed $24 / 7$.

The Phantom v310 digital video output is monitored at all times and recorded using digital video recorders (DVRs), which are also triggered by the GEN16t transient recorders. The DVR has a 1 -minute circular buffer with a $50 \%$ pretrigger. The Phantom v 310 cameras are configured in segmented-memory mode and they download each segment as data is gathered, while continuing to record if a qualified trigger is received. The Phantom v310 segment is set to an acquisition window of at least twice the time acquisition window of the GEN16t, with a $50 \%$ pretrigger. The reason for this is that the v310 will not acknowledge a subsequent trigger, whose pretrigger overlaps with the posttrigger of a previous segment. With this timing setup, we eliminate any potential blind spots in the acquisition of the highspeed video.

A differential-time-of-arrival technique is used with the B-dot and D-dot sensors to locate the attachment point. The downlead currents are used to estimate the intensity of the strike. The Phantom v310 cameras are used to locate the attachment point.

\section{ICLRT TESTS}

The International Center for Lightning Research and Testing (ICLRT) at Camp Blanding, Florida, is a 100-acre test facility, located between Gainesville and Jacksonville and operated by the University of Florida (UF) Lightning Research Laboratory. It has been continuously conducting triggered-lightning experiments since 1994.

During the summers of 2009 and 2010, instrumentation was deployed and tested at the ICLRT.

\subsection{Experiments}

Figure 7 shows a portion of the ICLRT where the deployed instrumentation was installed, as well as the instrumentation locations for the summer of 2009.

Throughout the summer of 2009, multiple configurations were set up to test both a single GEN16t and a pair (master/slave) of GEN16t transient recorders. In both cases, the master transient recorder was connected via single-mode optical fibers to multiple Isolated Digitizers 7600 installed in the field (inside EMI-shielded instrumentation boxes and powered by batteries [Figure 3]) close to each of the sensors used. Overall, a total of nine B-dot sensors (three 3-sensor $\mathrm{dH} / \mathrm{dt}$ stations at $\mathrm{S} 1, \mathrm{~S} 2$, and $\mathrm{S} 3$, respectively), five $\mathrm{D}$-dot sensors (five single-sensor $\mathrm{dE} / \mathrm{dt}$ stations at $\mathrm{S} 1, \mathrm{~S} 2, \mathrm{~S} 3$, $\mathrm{S} 4$, and atop the tower launcher), and one Phantom camera $^{2}$ (v10 at the main office, triggered by the ICLRT staff) were deployed and tested at the ICLRT and were subject to triggered and natural lightning strike events where vast amounts of data were collected. Transient recorders and high speed video cameras are synchronized using IRIG-B, provided by the ICLRT.

\footnotetext{
${ }^{2}$ Two additional Phantom v310 cameras were installed (in the front office) late during the summer with their trigger-in coming from the master transient recorder. Unfortunately after these cameras were installed, there was no more triggered lightning activity, so during 2009 , these cameras did not acquire any data.
} 


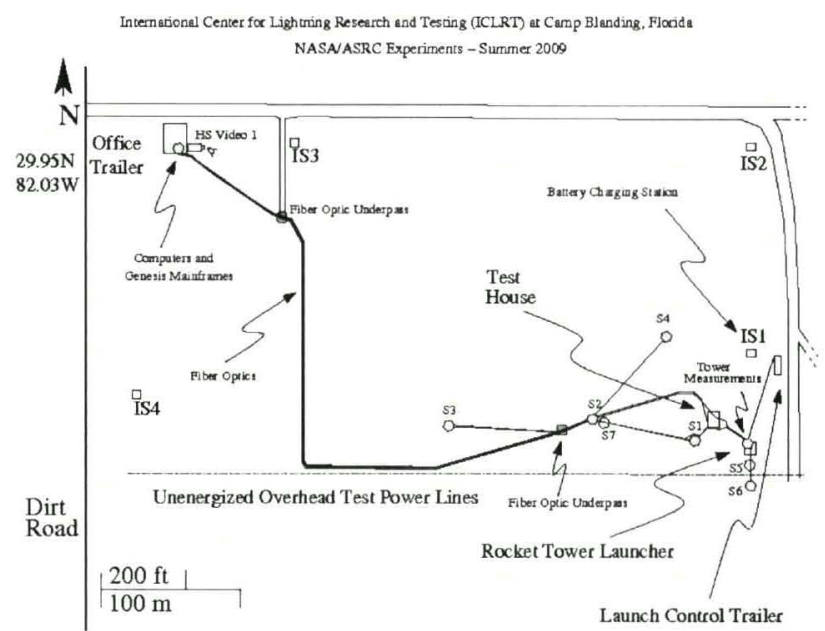

Figure 7. Outline of a portion of the ICLRT showing NASA/ASRC instrumentation (identified with smaller font) location during the summer of 2009.

For every classic rocket-triggered lightning event, the strike location is known (rocket tower launcher), which offers a paramount advantage when evaluating strike location algorithms. For this matter, $\mathrm{dH} / \mathrm{dt}$ data (threeaxis measurements) was acquired at distances of approximately $40 \mathrm{~m}, 130 \mathrm{~m}$, and $225 \mathrm{~m}$ from the rocket tower launcher; $\mathrm{dE} / \mathrm{dt}$ data was acquired at distances of approximately $5 \mathrm{~m}, 40 \mathrm{~m}, 90 \mathrm{~m}, 130 \mathrm{~m}$, and $225 \mathrm{~m}$ from the rocket tower launcher; and incident lightning current was recorded (at the base of the rocket tower launcher), whereas the high-speed video camera (Phantom v10) was installed $440 \mathrm{~m}$ from the rocket tower launcher. Apart from acquiring data from classic rocket-triggered lightning, there is also data collected for one unintended altitude rocket-triggered lightning event and several natural lightning strikes.

One main computer was connected to the GEN16t transient recorder via a GB Ethernet switch, powered from a UPS, housed at the office trailer (see Figure 7). In addition, a second computer was controlling (and collecting the data of) two Phantom v310 cameras. The trigger for these two cameras came from the GEN16t transient recorder, and all the communication and data transfer occurred via fiber-optic cables. These two cameras were intended to be installed in the field.

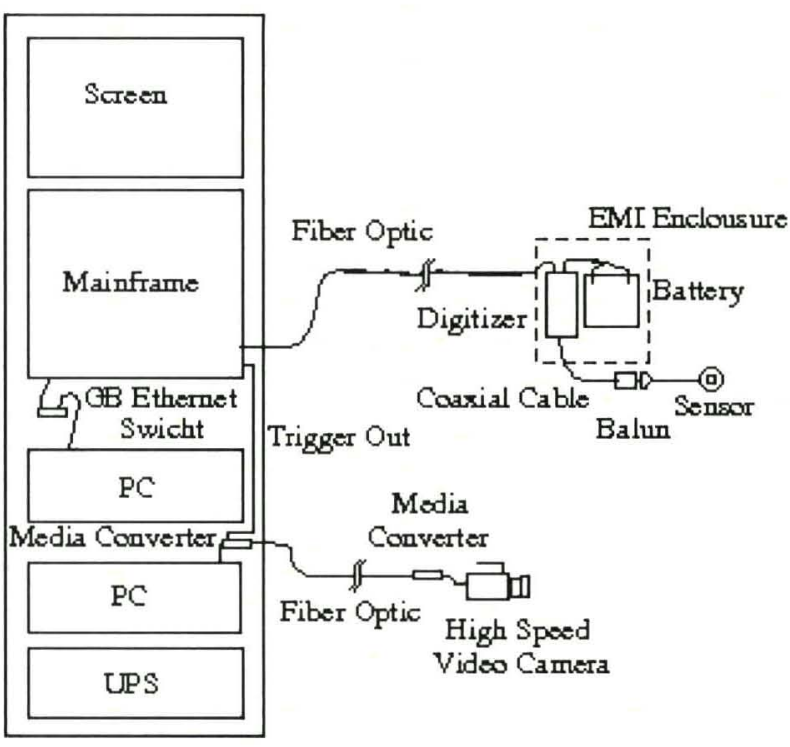

Figure 8. General instrumentation sketch.

When the summer 2009 tests started, a Phantom v10 high-speed camera was taken to the ICLRT. This camera was manually triggered by the ICLRT staff, and it was controlled by a laptop.

Figure 8 shows the general instrumentation setup. Each single-mode fiber-optic pair connected to the transient recorder (or mainframe GEN16t) was connected to a digitizer/sensor pair (see Figure 9 and Figure 10) recording 18 measurements simultaneously.

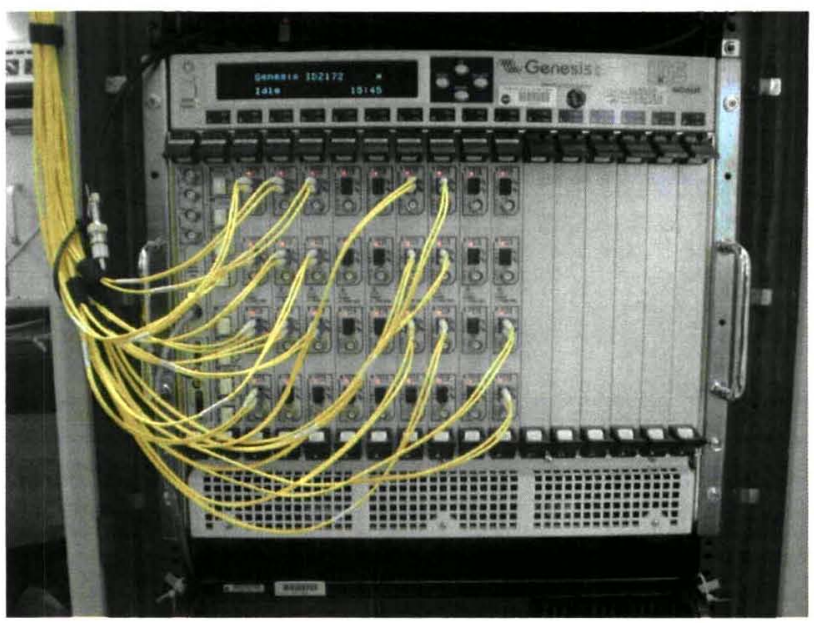

Figure 9. Transient recorder (GEN16t) with 18 singlemode fiber-optic links (May 22, 2009). 


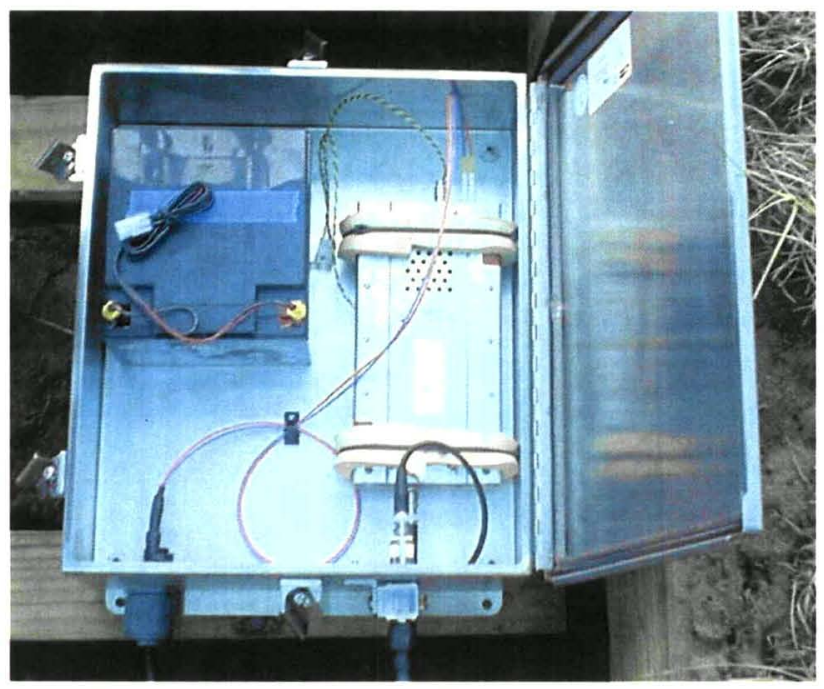

Figure 10. EMI instrumentation box with a digitizer connected to a battery and single-mode fiber-optic link.

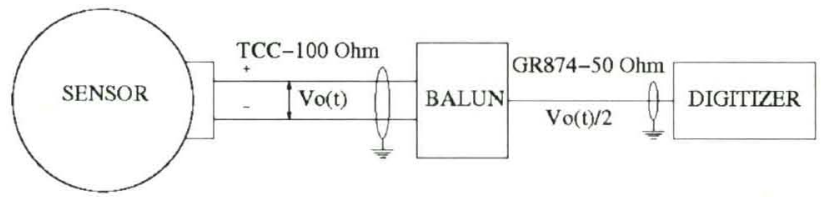

Figure 11. Connection diagram of a differential-mode balun with a free-field sensor.

\subsection{Experiments}

The 2010 experiments are an extension of the 2009 experiments, for which many measurements stay the same. The most significant differences are the installation (and instrumentation) of a scaled-down $(1 / 20)$ model of the new lightning protection system at LC39B and the addition of a mobile rocket launcher setup by the newly constructed, scaled-down LC39B lightning protection system.

Figure 12 shows a portion of the ICLRT (where the instrumentation was deployed in the spring of 2010) and indicates the instrumentation location, whereas Figure 13 shows, in more detail, the new, scaled-down lightning protection system. The downleads' current to ground was measured with Pearson Electronics Current Monitors, model 8525 . The sensors have a sensitivity of $0.01 \mathrm{~V} / \mathrm{A}$, maximum current rating of $50 \mathrm{kA}$, and a low and high cut-off frequency of $15 \mathrm{~Hz}$ and $15 \mathrm{MHz}$, respectively.

There was one (master) GEN16t transient recorder connected (via single-mode optical fibers) to several Isolated Digitizers 7600 installed in the field. Overall, a total of six B-dot sensors (two three-sensor $\mathrm{dH} / \mathrm{dt}$ stations at S2 and LPS, respectively), nine D-dot sensors (nine single-sensor dE/dt stations at S1, S2, S3, S4, S7, S8, S9, LPS, and atop the tower launcher) and three Phantom cameras (one v10 triggered by the ICLRT staff and two v310 triggered by the transient recorder, all at the main office). Transient recorders and high-speed video cameras are synchronized using IRIG-B, provided by the ICLRT.

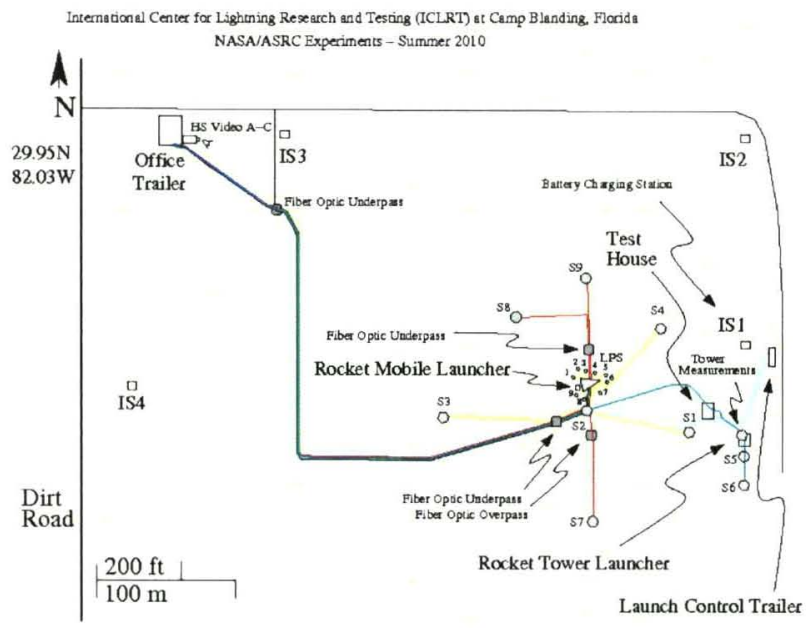

Figure 12. Outline of a portion of the ICLRT, showing NASA/ASRC instrumentation (identified with smaller font) location during the summer of 2010 .

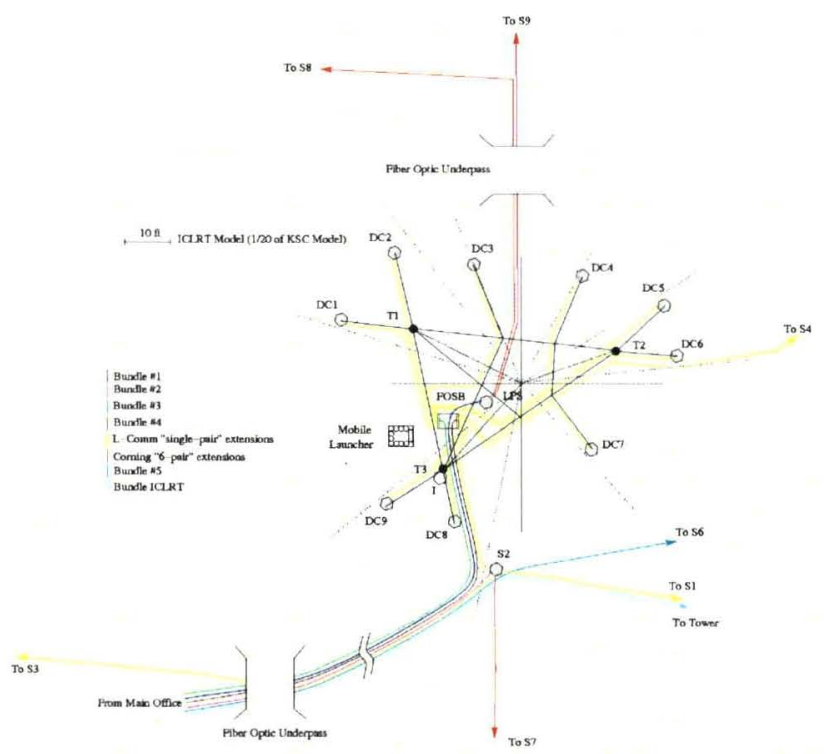

Figure 13. Zoom-in on the scaled lightning protection system built at the ICLRT for the 2010 experiments. 


\section{CONCLUSIONS}

A comprehensive lightning instrumentation system is presented for the new lightning protection system at LC39B at the Kennedy Space Center, Florida. The instrumentation system uses state-of-the-art transient recorders monitoring a multitude of current, B-dot, and D-dot sensors at various points of LC39B to estimate the strike intensity and the attachment point. High-speed video cameras are also used to monitor most of LC39B and to locate the attachment point.

\section{REFERENCES}

[1] C. T. Mata, V. A. Rakov, "Evaluation of lightning incidence to elements of a complex structure: a Monte Carlo approach," International Conference on Grounding and Earthing \& $3^{\text {rd }}$ International Conference on Lightning Physics and Effects (Ground 2008; $3^{\text {rd }}$ LPE), Florianopolis, Brazil, November 2008. 